\title{
EFFICIENT SOLUTION ALGORITHMS FOR HIGH-ACCURACY CENTRAL DIFFERENCE CFD SCHEMES
}

\author{
B. Treidler, J.A. Ekaterineris and R.E. Childs \\ Nielsen Engineering \& Research, Inc. \\ Mountain View, CA, 94043
}

\begin{abstract}
Preliminary results are presented from application of implicit integration schemes to high-accuracy CFD methods. High-accuracy refers to spatial discretization methods which are optimized for spectral bandwidth, rather than order of accuracy based on a Taylor' series expansion. The results show that reductions of more than $90 \%$ in computational effort, as measured by CPU time, can be achieved. The reduction is relative to second-order methods for obtaining steady solutions to the same level of accuracy. In addition, a variety of time integration methods have been evaluated for their use in time-accurate and steadystate simulations with the high-accuracy central difference schemes.
\end{abstract}

\section{Introduction}

The ability of high-order and high-accuracy discretization schemes for CFD to better resolve flow physics has been demonstrated by many researchers, e.g., Rangwalla and $\mathrm{Rai}^{1}$ and Treidler and Childs. ${ }^{2}$ In previous work by the authors, ${ }^{2}$ it was demonstrated that high-accuracy centered discretization schemes for CFD allowed an increase of a factor of four in the grid spacing required for a given solution accuracy, relative to second-order methods. However, the savings in CPU time through the use of fewer grid points were almost completely offset by the inefficiency of the explicit solution methods used for time integration. The purpose of this work is to evaluate implicit solution methods for high-accuracy central difference schemes.

High accuracy, as used here, is not a synonym for high order. High-order accurate discretization schemes based on Taylor series expansions are designed to perfectly represent as high an order of polynomial as possible, e.g., a five-point method will represent a fourth-order polynomial exactly. For infinitely small errors, the n-point Taylor series discretization gives the smallest possible error for a given stencil size. However, infinitely small errors are rarely achieved in
CFD. A more useful criterion is to force the errors to be practically zero for as large a wave number as possible. High accuracy is defined here to mean discretization schemes where the wave number below which a scheme does not exceed a given error level is maximized. The optimized compact schemes described by Lele $^{3}$ are another example of optimized methods.

The motivation for using high-accuracy/order schemes is to reduce the computational effort required to obtain a given level accuracy for a flow of interest. The ability of these schemes to reduce the number of grid points required for accurate solutions by orders of magnitude has been demonstrated. ${ }^{2}$ What remains is to develop efficient solution methods for steady and unsteady flows which will translate the orders of magnitude reductions in grid points into orders of magnitude reduction in CPU time required to obtain an accurate solution.

Solution algorithms for both steady and unsteady flows are considered in this work. Steady flow solution algorithms can be efficiently extended to unsteady cases through the use of dual timestepping. ${ }^{4,5}$ Dual time-stepping is a well-established technique which can be used for time-accurate solutions with high spatial accuracy methods.

In this paper, the properties of a variety of implicit solution methods are briefly described. The properties of these methods are then evaluated through the use of two simple problems. Finally, some results from an improved version of the high-accuracy CFD code from Treidler and Childs ${ }^{2}$ are presented to show dramatic reductions in the required CPU time for accurate solutions, relative to typical second-order methods. These reductions have been achieved despite using second-order implicit solver, rather than an implicit solver which is matched to the order of the explicit residuals. 


\section{Description of Solution Algorithms}

Numerical approximations to the nonlinear Euler equations give rise to dispersive errors. For central difference schemes, the dispersive errors are contributed mostly by the third derivative terms of the modified equation. It is, therefore, natural to use algorithms which have spatial order of accuracy four or higher to achieve better resolution and better dispersive properties required for long time integration. Implicit time integration with high-order accuracy in space can be obtained with direct solvers and application of GMRES methods. A list of possible time integration schemes for the Euler and thin-layer Navier-Stokes equations includes:

1. Explicit and implicit Runge-Kutta methods.

2. The Beam-Warming approximate factorization scheme. $^{6}$

3. The diagonalized implicit approximate factorization (DIAF) scheme. ${ }^{7}$

4. High spatial accuracy versions of implicit schemes 2 and 3.

5. Unfactored algorithms. ${ }^{8,9}$

6. Implicit Factorized Schemes which utilized compact spatial differencing

Explicit time integration with Runge-Kutta methods can provide low dispersion characteristics. ${ }^{10}$ Global high-order spatial accuracy is obtained when the residual term is evaluated with a high-accuracy scheme. However, explicit methods have stability limitations on time step size. For turbulent flow calculations, which require very fine meshes, and time dependent problems with long integration times, the large number of time steps required result in large computing times.

Implicit time integration methods are the alternative to explicit methods. An implicit method which has been successfully implemented in many current CFD codes is the alternating direction implicit (ADI) Beam-Warming ${ }^{6}$ algorithm. This method is second-order accurate in space and time. It is an approximately factorized algorithm and requires the inversion of block tridiagonal matrices for the sweep along each coordinate direction. Internal subiterations may be used during time advancement of the solution from time level $n$ to the new time level $n+1$ with physical time step $\Delta \mathrm{t}$ in order to eliminate linearization and factorization errors, and errors arising from employing lower order space discretization in the implicit operators. The error introduced by the approximate factorization is proportional to the CFL numbers along the transformed coordinate directions and imposes a limitation on the time step size $\Delta \mathrm{t}$.

The spatial order of accuracy of the implicit operators of the Beam-Warming algorithm can be increased to fourth order at the expense of increasing the bandwidth of the block matrix to five, which will make the implicit time integration computationally intensive.

Pulliam and Chaussee ${ }^{7}$ developed a diagonal implicit approximate factorization (DIAF) scheme which requires only scalar tridiagonal matrix inversions. The operation count and the memory space required for the diagonalized algorithm are dramatically decreased relative to the Beam-Warming algorithm. However, the diagonal algorithm is only first-order accurate in time. Second-order accuracy in time can be achieved by using a dual time step iteration scheme ${ }^{11}$.

The accuracy of the steady-state solution obtained with the DIAF algorithm is determined by the accuracy of the right hand side residual term. It is possible to increase the spatial accuracy of the method just by using high-accuracy methods to evaluate the explicit residual and retaining the low-order implicit algorithm. ${ }^{12}$ Alternatively, the spatial accuracy of both the explicit and implicit terms can be increased. Fourth-order accurate implicit central-difference operators for the DIAF method require inversions of scalar pentadiagonal matrices. These inversions are computationally efficient.

Another possible type of solution algorithm for the Euler equation uses compact differencing in ADI schemes. Examples are presented by Abarbanel and Kumar $^{13}$ and Ekaterineris. ${ }^{14}$ These schemes provide good dispersion characteristics for long time integration at a small increase in the computational effort for the inversion of the implicit operators relative to second-order methods. Existing codes can be adapted to use these algorithms with little modification.

In the rest of this paper, results from sample calculations will be presented. The dispersive properties of high-order implicit schemes and their convergence rates will be compared. Finally, results from a full CFD code, which show large savings in CPU time relative to a second-order code, will be presented.

\section{Results}

It is necessary to evaluate the time-accuracy, efficiency and accuracy of any solution method. For 
time-accuracy, a good test is to evaluate the dispersion characteristics of the methods on a linear model problem. Numerical solution of a linear problem reveals the potential of the methods while avoiding complicating factors, such as artificial dissipation, boundary conditions, and grid metric accuracy.

For solution efficiency and accuracy, three sets of results are presented below. First, the convergence properties of several schemes, as used to determine the flow around a NACA-0012 airfoil, are evaluated. Results are shown for both subsonic and transonic flow. Next, a direct comparison is made for the CPU time required to obtain solutions for inviscid flow around a circular cylinder. Finally, the same code which was used for the cylinder flow, is used to calculate flow around a NACA-0012 airfoil and the accuracy is compared with low-order methods.

\section{Dispersive Properties of Time Integration Schemes}

The simple model problem is convection of a Gaussian density pulse. The pulse is described by $\rho=\rho+\rho^{\prime}=\rho_{\circ}+\exp \left[-\log 2\left(\left(\mathrm{x}-\mathrm{x}_{\circ}\right) / \mathrm{c}\right)^{2}\right]$. The length scale $\mathrm{c}$ is 10 . This is a purely linear problem, so artificial dissipation is not required. This eliminates one of the difficulties in evaluating central difference methods for nonlinear problems, the necessity of using artificial dissipation to prevent numerical oscillations.

Central difference schemes are symmetric and nondissipative. As a result, the dispersion characteristics of each scheme can be easily studied. Dispersion characteristics are very important for the application of the methods to unsteady flow problems where long time integration is required.

A uniform grid was used for the simulations. The spacing produced 20 points across the pulse. This is sufficient to resolve the pulse with both second- and fourth-order methods.

The density pulse was convected for 50 nondimensional unit lengths. The pulse shape obtained from the computations with five methods are compared with the exact solution in Figure 1. Of the five schemes in Figure 1, four used compact spatial differencing: the fourth-order accurate in space, block tridiagonal algorithm of Ekaterineris ${ }^{14}(\mathrm{C}-1)$, the fourth-order accurate algorithm of Abarbanel and $\operatorname{Kumar}^{13}$ (C-2), the diagonal fourth-order compact algorithm of Ekaterineris ${ }^{14}$ (C-3), and the explicit four-stage Runge-Kutta method (C-4). For curve C-4, the inviscid fluxes were evaluated with fourth-order accurate compact differences. The three nondiagonal compact schemes produced results which were identical to plotting accuracy and show good agreement with the exact solution. The solution obtained with the first-order accurate in time and fourth-order in space compact diagonalized algorithm, (C-3), shows a little dispersion compared to the exact solution. The pulse shape obtained by a solution with the standard second-order accurate in space and time block tridiagonal Beam-Warming algorithm with fourth-order explicit central difference residual evaluation, (C-5), shows the largest variation from the analytic solution due to the dispersive and diffusive nature of the second-order implicit part of the scheme.

\section{Convergence Rates for Implicit Solution Algorithms}

For steady-state solutions, the numerical diffusion of the Beam-Warming and DIAF schemes is not a serious handicap to the accuracy of the solution. This is because the final solution is determined completely by the accuracy of the explicit residual terms. What will most certainly be affected by the low-order nature of the implicit schemes is the rate of convergence.

The diagonalized algorithm of Pulliam and Chaussee, ${ }^{7}$ was used to compute steady-state inviscid flow solutions over a NACA-0012 airfoil. For steady-state computations, first-order accuracy in time is sufficient, and it is unnecessary to perform time integration with the second-order accurate in time, dual time-stepping, diagonalized algorithm.

Convergence rates for subsonic and transonic flow solutions obtained by three algorithms are shown in Figures 2 and 3, respectively. The three shemes are: the standard, second-order accurate in space diagonalized algorithm (R2-L2) of Pulliam and Chaussee, ${ }^{7}$ the fourth-order accurate in space version of the diagonalized algorithm (R4-L4), and the compact, fourth-order accurate algorithm (R4c-L4c) of Ekaterineris. ${ }^{14}$ The (R4-L4) algorithm is a straightforward extension of the (R2-L2) algorithm and evaluates the residual term with fourth-order accurate, explicit, five-point stencil, central-difference formulas and performs the differentiations of the implicit operators also by using the same fourth-order accurate central differences. Fourth-order accuracy for the implicit operators is obtained at an increase of the computing cost because, in addition to the higher operation count required for the evaluation of the spatial derivatives with fourth-order accuracy, a pentadiagonal matrix inversion is also required. The fourth-order accurate scheme (R4-L4) has a slightly better convergence than the standard (R2-L2) algorithm. The convergence rate obtained with the 
compact, fourth-order accurate algorithm (R4c-L4c) of Ekaterineris $^{14}$ is also shown. The fourth-order compact algorithm achieves a better convergence rate at a small increase in the computing cost resulting from the computation of the residual term with compact finite differences. The implicit inversion of the compact (R4c-L4c) algorithm is obtained at the same computing cost as the standard algorithm because it involves tridiagonal matrices.

Convergence rates obtained for the computation of inviscid transonic flow at freestream speed $\mathrm{M}=0.8$ and $\alpha=0.1 \mathrm{deg}$. over the NACA-0012 airfoil with the (R2-L2) and (R4c-L4c) algorithms are shown in Figure 3. The trends of the subsonic flow computation carry over to the transonic flow. A solution with the R4-L4 schemes is not shown because it did not show improved convergence to steady state.

Comparison of Accuracy and Computational Effort for DIAF and a High-Accuracy Steady-State Scheme

In the results below, comparisons will be made between OVERFLOW ${ }^{15}$ and the OVERHI code of Treidler and Childs. ${ }^{2}$ OVERFLOW is a second-order accurate code with many options for time-stepping. In this case it was used with a variant of the DIAF scheme. Multigrid acceleration was used in the OVERFLOW calculations when it provided significant benefits. OVERHI is a version of OVERFLOW in which high-accuracy spatial discretization schemes (up to eight-point stencils) have been implemented for the evaluation of the explicit residuals. The artificial dissipation used in OVERHI also uses an eight-point stencil.

For subsonic flows, the implicit Beam-Warming approximate factorization method, as implemented in OVERFLOW, was found to converge quickly to a steady-state solution when used with five-point stencil high-accuracy methods for evaluation of the explicit terms which determine the physics and the eight-point stencil explicit artificial dissipation. Second-order artificial dissipation is used for the implicit operator. Pulliam $^{16}$ has shown that it is critical to match the order of the implicit and explicit dissipation models. This has not been done in OVERHI. Thus, the OVERHI results for CPU time are far from optimized and represent a lower bounds on the potential savings from using high-accuracy methods.

In the results below, comparisons of CPU times required to obtain a certain level of accuracy are only relevant for the inviscid cylinder flows. For the airfoil flows, the use of low-accuracy c-grid boundary conditions prevents OVERHI from showing high- accuracy convergence of force coefficients. The CPU times presented for OVERHI include the CPU time for running OVERFLOW to convergence on the same grid. OVERHI solutions were generated starting from the converged OVERFLOW solutions.

The contour plots shown below assume a linear variation between grid points. The contour lines do not account for the high accuracy of OVERHI. Consequently, there are cases below where the OVERHI solution on a coarse grid is equivalent to that on a finer grid, but the plots show differences in the contour lines away from grid points on the coarse grid.

\section{Inviscid Flow Past a Cylinder}

Inviscid flow past a cylinder was simulated at $\mathrm{M}=0.2$. Calculations were performed on grids with arc-spacings, in radians, of $\pi / 8, \pi / 16, \pi / 32, \pi / 64$, and $\pi / 128$. The grid is a semicircle because symmetry boundary conditions are used. The grid extends out thirty cylinder diameters, which is the distance that was found to eliminate the effect of the characteristic far-field boundary condition on the force coefficients for transonic flow. A semicircle grid was used to save time, but the computed drag coefficient was found to be the same as when the whole flow was simulated for both OVERFLOW and OVERHI. The grid spacing in the radial direction is set equal to the grid spacing in the circumferential direction.

Contour plots of pressure from solutions on different grids computed with OVERFLOW and OVERHI are shown in Figures 4 and 5, respectively. The OVERFLOW contours vary in a manner consistent with a second-order accurate method, i.e., the shift in contours decreases by a factor of four each time the grid spacing is halved. The OVERHI results do not vary with grid spacing except for some small differences on the downstream side of the cylinder. The flow is resolved on even the coarsest grid shown. The $\pi / 16$ grid has a minimum wallnormal spacing of $\Delta \mathrm{r} / \mathrm{R} \sim 0.2$. OVERFLOW was never intended for use with such inadequate grid resolution but OVERHI produces results on this grid which are comparable to OVERFLOW's solution on a grid eight times finer. OVERHI and OVERFLOW do converge to the same solution on fine grids.

Figures 4 and 5 are a convincing demonstration of the ability of high-accuracy methods to accurately simulate flows on coarse grids. However, the motivation for using high-accuracy methods is to obtain accurate solutions with less CPU time, not fewer grid points. Figure 6 presents results for error in 
the computed drag coefficient, $\mathrm{C}_{\mathrm{D}}$, versus CPU times required for convergence. Since this is an inviscid subsonic flow, there should be no drag and $C_{D}$ is a valid measure of the error in the numerical solution. This figure shows that the OVERHI solution on the $\pi / 16$ grid is almost as accurate as the OVERFLOW solution on the $\pi / 128$ grid. OVERHI requires only $2 \%$ of the grid points and $3 \%$ of the CPU time required by OVERFLOW for the same level of accuracy. These are significant savings and are for a two-dimensional problem. Larger savings would occur in three dimensions.

The results in Figure 6 are for grids with radial spacing at the cylinder surface which is the same as in the circumferential direction. When the same series of runs was performed for grids where the initial radial spacing was $10 \%$ or $30 \%$ of the circumferential spacing, similar results were found. The results above are typical and no cases were found where OVERFLOW performed better than this relative to OVERHI.

Subsonic-Inviscid Airfoil Calculations

The final set of results for the high-accuracy methods are for two-dimensional inviscid flow over a NACA-0012 airfoil.

The grids used in the calculations are C-type grids which extend 30 chord lengths away from the airfoil surface. The grids used are classified by the minimum grid spacing on the airfoil surface, $\mathrm{ds}_{\min }$. The grid spacing varies by a factor of two on the surface, so that a grid with $\mathrm{ds}_{\min }=0.01$ chord lengths has a maximum spacing on the airfoil surface of 0.02 chord lengths. The spacing normal to the surface is $1 / 2$ of $\mathrm{ds}_{\text {min }}$.

A high-accuracy C-cut boundary condition has not been implemented in OVERHI. This degrades the global code order of accuracy to be the same as OVERFLOW for c-grids. However, it is still possible to compare the solutions to see how OVERHI performs.

Simulations were performed for subsonic inviscid flow at an angle of attack of 10 degrees and a freestream Mach number of 0.2. Figures 7 and 8 show contours of Mach number for OVERFLOW and OVERHI, respectively. The main points to note are that OVERFLOW, even for the finest grid, has a significant "boundary layer" for the Mach number, while OVERHI has eliminated this boundary layer for the two finest grids. The "boundary layer" for the Mach number is actually a boundary layer in temperature, which increases the Mach number near the surface. There are no "boundary layers" for pressure or fluid velocity. OVERHI seems to have fully resolved the flow for $\mathrm{ds}_{\min }=0.0025$. The OVERHI solutions are accurate, even for the coarsest grid, everywhere except the upper surface of the airfoil. Except on the upper surface of the airfoil, the greatest variations between the $\mathrm{ds}_{\min }=0.01$ contours and the contours for finer grids result mainly from the plotting method.

Because of the low-order c-grid boundary condition, OVERHI does not exhibit the high-order convergence of force coefficients which was seen for the inviscid cylinder flows. However, OVERHI is more accurate than OVERFLOW for a given grid spacing.

Other configurations

OVERHI has also been used for laminar thin-layer Navier-Stokes calculations, three-dimensional calculations, and transonic flow calculations, but comprehensive grid refinement studies have not been carried out.

\section{Conclusions}

From the results presented above, it is clear that high-accuracy methods have the potential for reducing CPU time and grid size requirements for CFD calculations by at least two orders of magnitude for two-dimensional calculations and by a higher ratio for three-dimensional calculations. Savings of $98 \%$ in CPU time have been demonstrated for a twodimensional subsonic case with an implicit scheme which was not modified to account for spatial highaccuracy. This represents a lower limit on the potential savings from high-accuracy spatial discretizations.

It is not possible to conclude which of the schemes evaluated in the first results section of this paper is most appropriate for use in CFD. The dispersion characteristics of all of the nondiagonal schemes were comparable. The combination of dual time-stepping with the diagonal schemes remains to be evaluated. For convergence rate, the compact schemes seem to have a small advantage.

\section{Acknowledgements}

The authors gratefully acknowledge support for this work received from the U.S. Air Force Wright Laboratory/FIMC (Contract F33615-97-C-3001) and the U.S. Army Research Office (Contract DAAG5597-C-0029). 


\section{References}

1. Rangwalla, A. A. and Rai, M. M., "A MultiZone High-Order Finite Difference Method for the Navier-Stokes Equations," AIAA Paper 95-1706-CP, 1995.

2. Treidler, E. B. and Childs, R. E., "HighAccuracy Spatial Discretization Schemes for CFD," AIAA Paper 97-0541, 1997.

3. Lele, S. K., "Compact Finite Difference Schemes with Spectral-Like Resolution," J. Comp. Phys., Vol. 103, No. 1, 1992, pp. 16-42.

4. Merkle, C.L. and Athavale, M., "Time Accurate Unsteady Incompressible Flow Algorithm Based on Artificial Compressibility," AIAA Paper 87-1137, 1987.

5. Venkatakrishnan, V. and Mavriplis, D.J., "Implicit Method for the Computation of Unsteady Flows on Unstructured Grids," J. Comp. Phys., Vol. 127, 1996, pp. 380-397.

6. Beam, R. M. and Warming, R. F., "An Implicit Factored Scheme for the Compressible Navier-Stokes Equations," AIAA Journal, Vol. 16, No. 4, 1978, pp. 393-402.

7. Pulliam, T. H. and Chausse, D. S., "A Diagonal Form of an Implicit Approximate Factorization Algorithm," J. Comp. Phys., Vol. 39, No. 2, 1981, pp. 347-363.

8. Chakravarthy, S., "Relaxation Methods for Unfactored Implicit Upwind Schemes," AIAA Paper 84-0165, 1984.

9. Yoon, S. and Jameson, A., "Lower-Upper Symmetric-Gauss-Seidel Method for the Euler and Navier-Stokes Equations," AIAA Journal, Vol. 26, No. 9, 1988, pp. 1025-1026.

10. Hu, F.Q., Hussaini, M.Y. and Manthey, J.L., "Low-Dispersion and Low-Dissipation Runge-Kutta Schemes for Computational Acoustics," J. Comp. Phys., Vol. 124, 1996, pp. 177-191.

11. DeRango, S. and Zingg, D. W.,

"Improvements to a Dual-Time-Stepping Method for Computing Unsteady Flows," AIAA Journal, Vol. 35, No. 8, 1997, p. 1548.

12. Visbal, M.R. and Gaitonde, D.V., "High-Order Accurate Methods for Unsteady Vortical Flows in Curvilinear Meshes," AIAA Paper 98-0131, 1998.

13. Abarbanel, S. and Kumar, A., "Compact High-Order Schemes for the Euler Equations,"
15. Buning, P.G., Chan, W.M., et al., "OVERFLOW User's Manual- Version 1.6.be," unpublished NASA document.

16. Pulliam, T. H., "Artificial Dissipation Models for the Euler Equations," AIAA Journal, Vol. 24, No. 12, 1986, pp. 1931-1940.

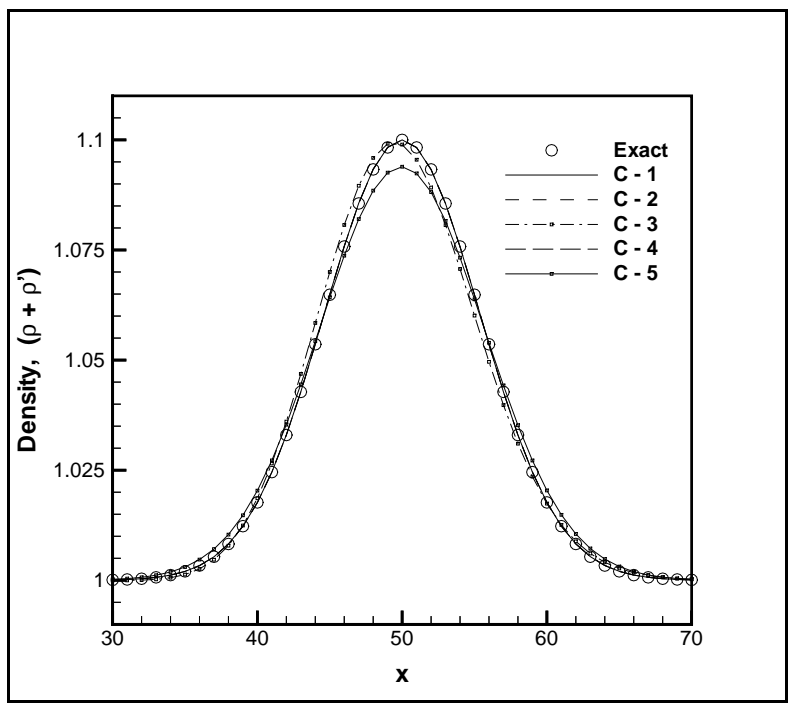

Figure 1 Comparison of the computed pulse shape with the exact solution for the convection of a density disturbance:

(C-1) 4th order, block tridiagonal, compact implicit.

(C-2) 4th order, Abarbanel-Kumar.

(C-3) 4th order, diagonal, compact, implicit.

(C-4) explicit R-K-4, 4th order compact right--hand side (rhs).

(C-5) Beam-Warming 2nd order in space implicit, 4th order accurate rhs. 


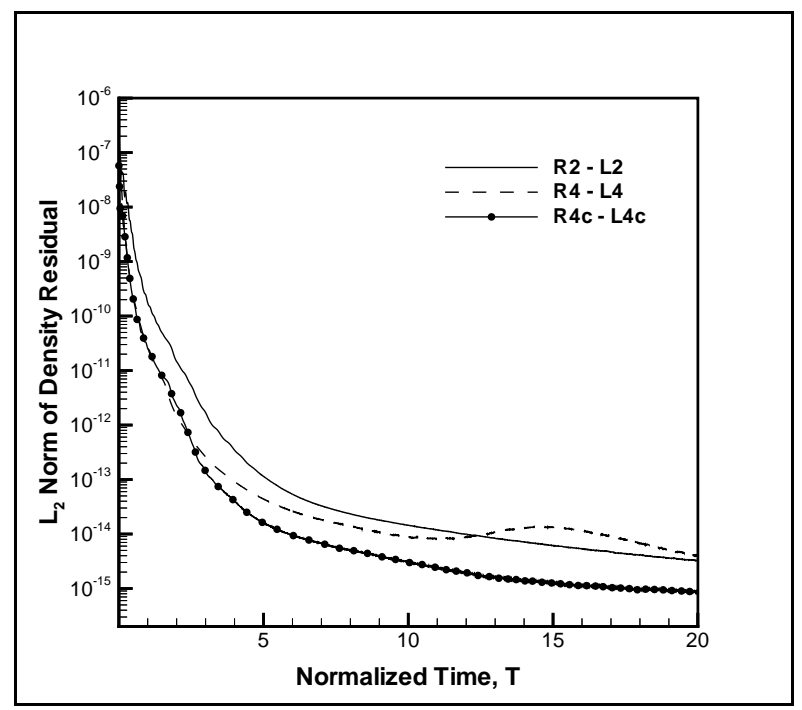

Figure 2: Convergence of the diagonalized algorithm shown by the $L_{2}$ norm of density residuals for inviscid flow over an airfoil at $M=0.3$ and $\alpha=10 \mathrm{deg}$. (R2-L2) Second order central-difference on both the right hand side (rhs) and left hand side (lhs). (R4-L4) Fourth order explicit central-difference (rhs) and (lhs) with pentadiagonal matrix inversion. (R4c-L4c) Fourth order explicit compact-difference (rhs) and (lhs) with tridiagonal matrix inversion.

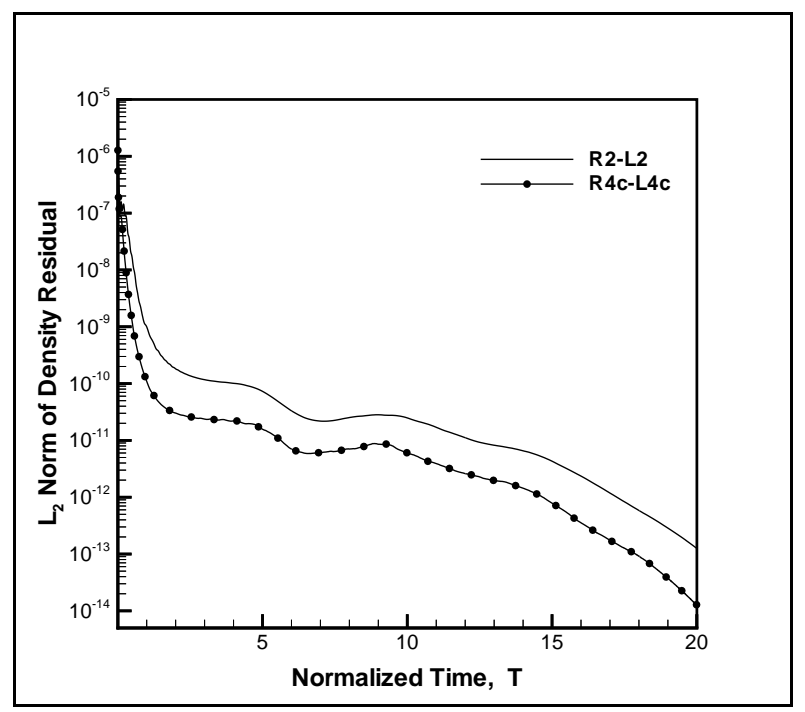

Figure 3: Convergence of the diagonalized algorithm shown by the $\mathrm{L}_{2}$ norm of density residuals for inviscid transonic flow over an airfoil at $\mathrm{M}=0.8$ and $\alpha=0.1$ deg. (R2-L2) Second order central-difference on both the right hand side (rhs) and left hand side (lhs). (R4c-L4c) Fourth order explicit compact-difference (rhs) and (lhs) with tridiagonal matrix inversion.

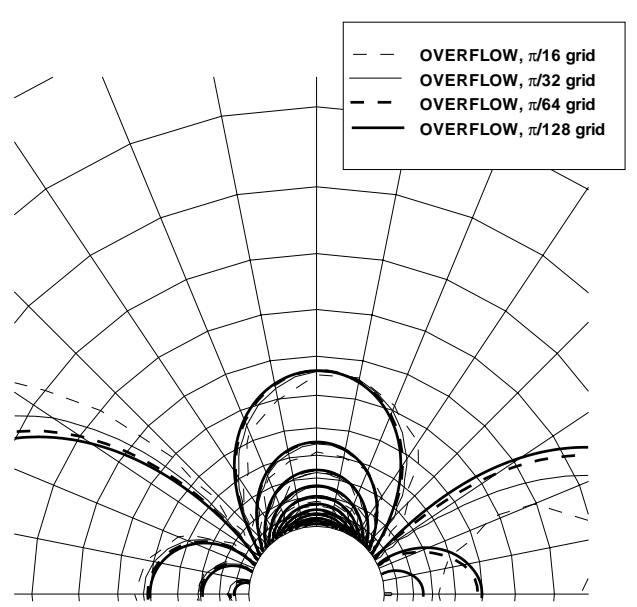

Figure 4: Computed pressure contours for inviscid flow past a cylinder at a Mach number of 0.2. Results from OVERFLOW are presented for grids with circumferential spacings of $\pi / 16, \pi / 32, \pi / 64$ and $\pi / 128$. Flow is from left to right and the grid lines are from the $\pi / 16$ grid.

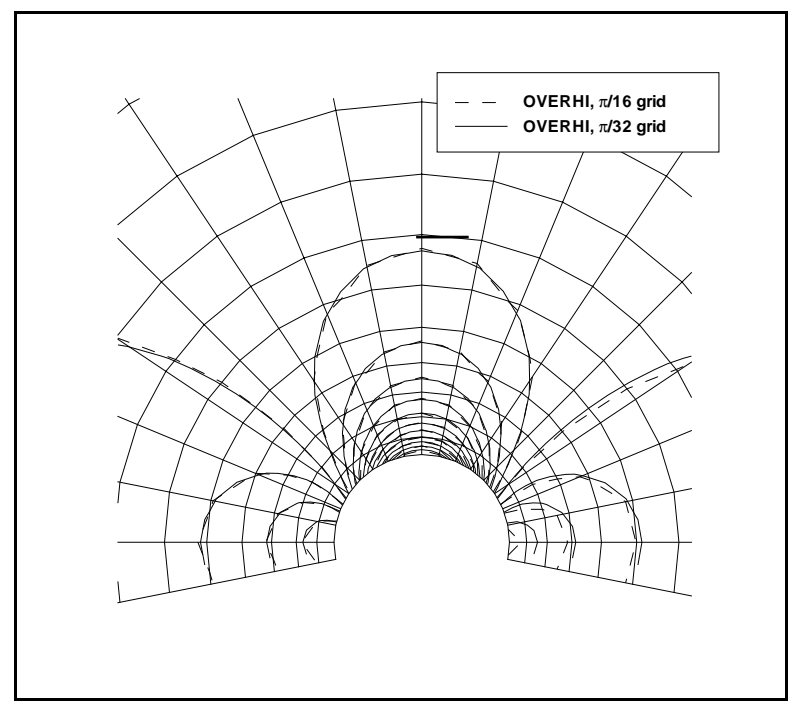

Figure 5: Computed pressure contours for inviscid flow past a cylinder at a Mach number of 0.2. Results from OVERHI are presented for grids with circumferential spacings of $\pi / 16$ and $\pi / 32$. Flow is from left to right and the grid lines are from the $\pi / 16$ grid. 


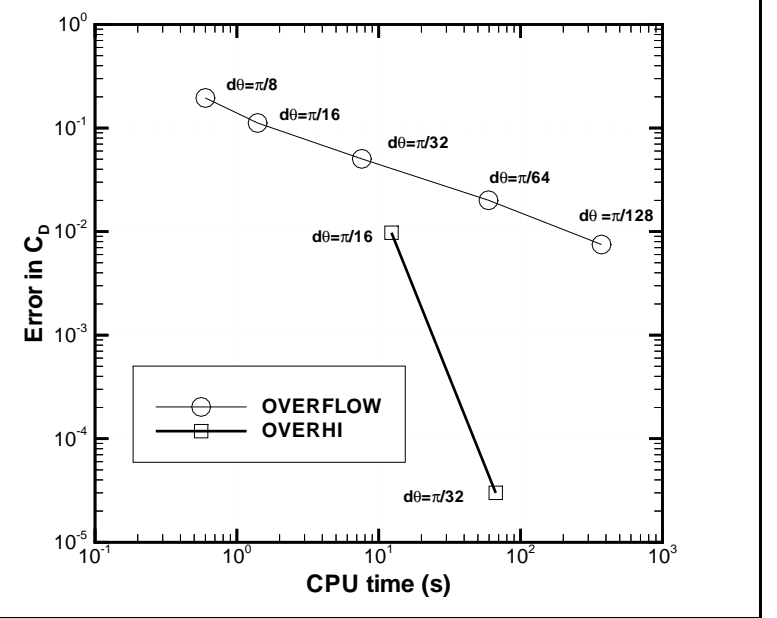

Figure 6: Error in $\mathrm{C}_{\mathrm{D}}$ is plotted versus CPU time on an HP C180 for OVERFLOW and OVERHI. The simulations are for steady inviscid two-dimensional flow past a circular cylinder at a Mach number of 0.2 .

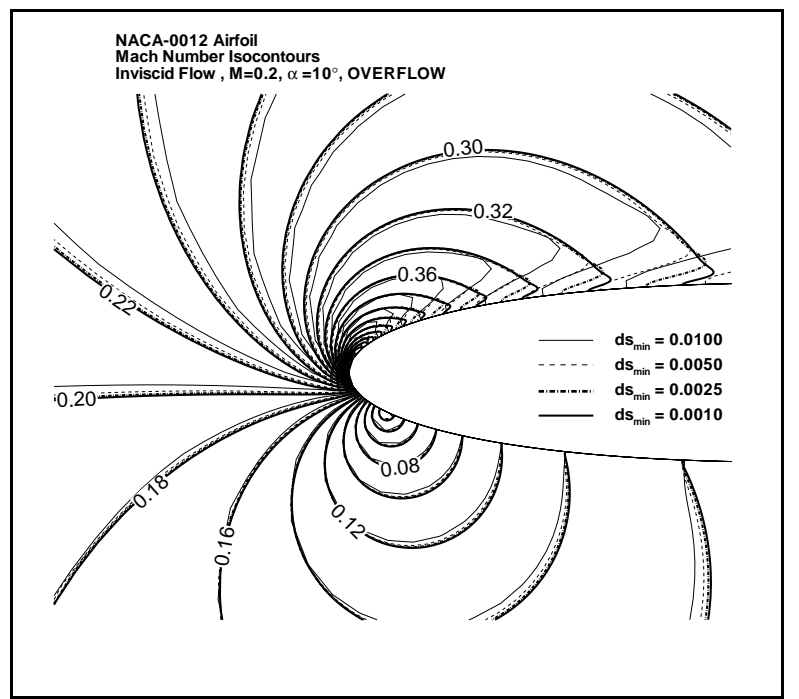

Figure 7: Isocontours of Mach number for inviscid flow past a NACA-0012 airfoil at a freestream Mach number of 0.2 and an angle of attack of 10 degrees. Results are from OVERFLOW for several different grids. $\mathrm{dS}_{\mathrm{min}}$ is the minimum grid spacing along the airfoil surface.

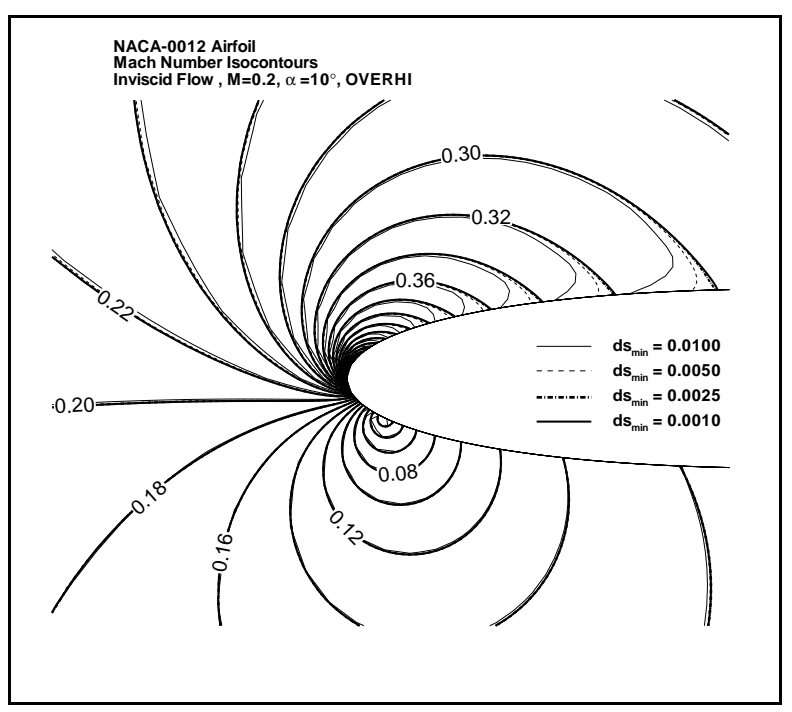

Figure 8: Isocontours of Mach number for inviscid flow past a NACA-0012 airfoil at a freestream Mach number of 0.2 and an angle of attack of 10 degrees. Results are from OVERHI for several different grids. $\mathrm{dS}_{\mathrm{min}}$ is the minimum grid spacing along the airfoil surface. 